\title{
Rock bumps due to the creation of a dislocation during deep mining
}

\author{
V. Doležel ${ }^{1} \&$ P. Procházka ${ }^{2}$ \\ ${ }^{1}$ University of Pardubice, Czech Republic \\ ${ }^{2}$ Czech Technical University in Prague, Czech Republic
}

\begin{abstract}
The safety of deep mines is sometimes threatened by sudden and hazardous failures of a coal face, as in the case of bumps, for example. It has been shown in many experimental studies that coal bumps are influenced either by stress, stiffness, and yield ability of surrounding rocks, or by dynamic effects associated with the failure of surrounding strata. In addition, bumps occurred in such parts of mines where there had been rapid stress changes over a short period of time. The dynamic effects associated with the failure of surrounding strata triggered bumps in these marginally stable seam structures. While it was not possible to evaluate the influence of mine stiffness directly, it was shown that coal bumps generally occurred in mines with uniaxial compressive strength and Young's modulus ratios (roof to coal) exceeding 3 to 5. In addition, bump-prone coal exhibited the potential for storing high horizontal stresses. Yielding of the immediate roof and floor reduced horizontal stresses and enhanced gradual failure of coal. A method is proposed to assess coal bumps in which stress analyses, in situ strength data, stiffness and strength ratios of roof to coal, and affected wave magnitude resulting from strata failure and mining experience are incorporated. A rheological model is involved to fulfill the time dependence of the phenomenon of bumps. It has namely been shown in the literature that the time plays a decisive role in the possible occurrence of rock bursts. In our case the static (stability) problem is solved and the dynamical influence of inertia forces is initiated after occurrence of bumps. This is not of any importance for us, as we suppose that the mine is far enough from other openings (other mines). In this way the stability problem with possible rheology (or creep) is studied. Keywords: rock bursts (bumps), scale modeling, coupled modeling, natural existence of occurrence of bumps, numerical model of contact problem.
\end{abstract}




\section{Introduction}

The paper deals with an application of coupled modeling in identification of dislocation occurring in coal mines threatening workers in the underground structures. The bumps can be induced for different reasons, one being the accumulation of energy in unpredictable dislocations. The measurement on site is very expensive and unreliable. One of the most reliable is physical modeling, which enables one to carry out parametric studies and after certain results from these models one can assess the most probable concentration of stresses. On the other hand, the stresses are measured in a very difficult way, so that numerical analysis should take place. In numerical analysis the contact problem in limit state estimation is based on data from physical modeling. The physical modeling seems to be the best for linear analysis. This is not the case of our study and large attempts have to be carried out to estimate the real behavior of the material. With support of physical modeling mathematical formulation and numerical treatment can lead to the danger of occurrence of bumps. In general, the large iteration should be used to solve the strongly nonlinear problem in both subdomains ranged in boundaries given by possible dislocations, and on the interfacial boundary (dislocations). In order to eliminate some principal directions of iteration the physical modeling is used and the numerical processes become bearable. A typical example from praxis verifies the theory based on back analysis.

Extensive studies were done by Elices et al., Haramy and McDonnell [3] and Haramy et al., [4], who showed a methodology on how to estimate the possibility of bumps. In [2] the authors tried to explain reasons for bumps based on a couple of experiments. The conditions for bumps in coal mines under a strong roof are described in [3], where possible bumps are analyzed and explained. A methodology for assessment of pillars in longwall mining is suggested in [5]. Scale modeling of rock bursts is described in [6]. The method and equipment mentioned in [6] are very powerful for illustration of bump evaluation, as the extrusion of coal grains into free space can be seen in a natural way.

Numerical modeling can be found in [7], for example, where the cracking is described by a cohesive zone method, which starts with Griffith and Barenblatt theories. A very promising method seems to be partition of unity, presented in [8], for example, where a theoretical explanation can be found, or in [9], where a direct application of the mentioned method to a cracking material is shown. Another method appropriate for description of cracking is called the manifold method; its advanced version is published in [10]. Discrete methods became very popular in describing nucleation of cracks and consequent occurrence of bumps. One of such method is denoted as free hexagons of statical particle flow code, [11]. Coupled modeling, consisting of implementation of results from experimental (scale) models into numerical computation, is proposed in [12]. It is based on involvement of eigenstrains or eigenstresses as design parameters in identification of material properties.

In this paper the most dangerous crack in a rock overburden of a coal seam is determined. 


\section{Experimental study}

The principal objective of the experimental and numerical model research was the clarification of mechanical behavior of the coal seam and a close and distant overburden of one selected seam in connection with the origin of rock bumps in the proximity of localization in the north Czech Republic.

The first phase comprised the simulation of exploitation of the seam under consideration. The extraordinarily difficult and exacting character of the problem required an unconventional approach to its solution. For this reason the physical model of equivalent materials was supplemented with a mathematical and a scale model. This combined approach was selected to make the individual partial methods mutually link up and complement each other to advantage and so enable a more comprehensive insight into the whole problem.

As some data required by mechanical properties, particularly those concerning the shear and triaxial strength, were not available (the required measurements had not been made in the given area), it was necessary to verify the strength characteristics of the rock mass in the environs of the principal crosscut by a contact problem. Experiments with models from physically equivalent materials provided a detailed stress distribution in rock environment (with the assumption of its ideally elastic behavior) and supplemented very appropriately the results of measurements made on physical models. Generally, two concepts of rock bursts are distinguished: The first assumes that a first bump occurs and then the dislocation appears as a consequent of weakened overburden. The second idea starts with the dislocation in the overburden and the bumps is an aftermath of the situation caused by the cracked dislocation. The latter case is considered in this study after obtaining the realistic material properties from measurements on site. Hence the appraisal of the relations and consequently the maintenance of the situation on site can be carried out. The geometry and possible failure in the neighborhood of the face of the long wall mining is depicted in Fig. 1, where also the source of dynamic propagation is shown.
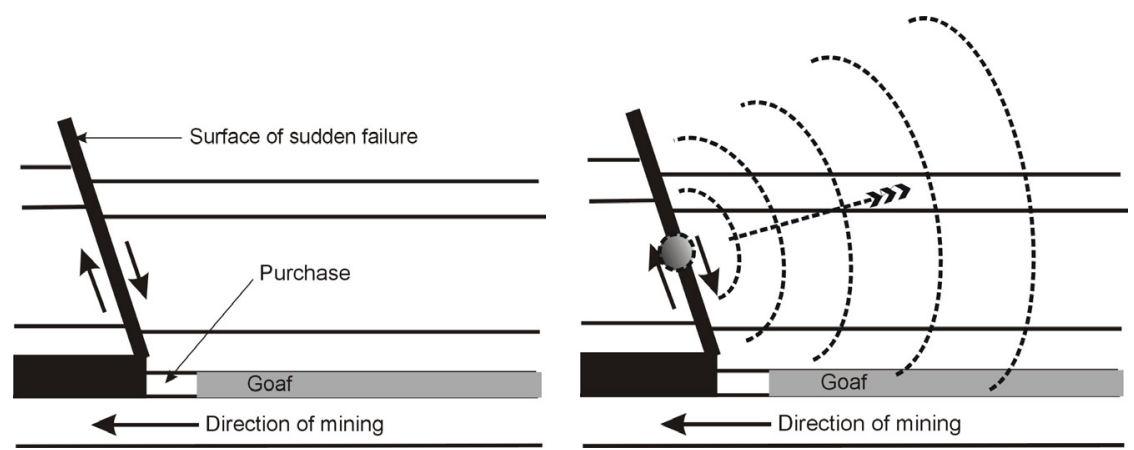

Figure 1: $\quad$ Static and seismic energy released after limit concentration at the face of the wall. 


\section{Verification of material parameters of rock environment}

To determine the characteristics of equivalent material used for physical model construction it was necessary to comply with the requirements of physical similarity between the model and the rock environment. However, the measurements required for this purpose were not made in the full extent. For this reason it was decided to verify and/or refine the selected shear parameters of the rock mass by the DEM in such a way that the results for the free hexagons fed by data from laboratory experiments had to comply with the results from beards build up in the beginning of the construction of the mines. It appeared that there were deep differences in these results and the numerical approach had to be basically improved.

Long term monitoring of the rock mass in crosscut environs has ascertained that its extraction does not impair ambient rock. Consequently the shear parameters not producing any extensive failure zone in the environs of the working may be considered as the basic shear strength of the rock mass. Under these assumptions the problems were formulated as follows:

1. The shear parameters were estimated on the basis of the rock strength (admissible values of the material parameters) in uniaxial compression and tension and literary data.

$$
\text { Alternative A : } \quad \begin{aligned}
c & =5.5 \mathrm{MPa} \\
\varphi & =35^{\circ} \\
\sigma_{+} & =5.0 \mathrm{MPa}
\end{aligned}
$$

where $c$ is the cohesion (shear strength), $\varphi$ is the angle of internal friction, and $\sigma_{+}$is the tensile strength.

2. The non-linear problem, based on the same shear strength assumptions, was solved to refine the convergence of the working.

3. The problem solved as another alternative, was based on the following strength parameters:

Alternative B:

$$
\begin{aligned}
& c=10 \mathrm{MPa} \\
& \varphi=65.5^{\circ} \\
& \sigma_{+}=4.1 \mathrm{MPa}
\end{aligned}
$$

The difference in the extent of failure zones should show the minimum shear strength of the rock mass. The solution of all problems assumed the initial stress state due to overburden weight and characterized by the value of the coefficient of the side pressure $K_{e}=0.54$. Physical non-linearity was concentrated on the dislocation. The program makes it possible to respect the different behavior of the materials in compression, in tension and after load relief.

Compressive strength is controlled in accordance with the first failure theory, i.e., by a comparison of the maximum shear stress with the shear strength of the respective material. This control is performed only if the octahedral normal stress $\sigma_{\text {oct }}$ is a shear stress. In the opposite case the control concerns shear according to the generalized Mohr theory of failure expressed in octahedral stresses. 


\section{Solution of underground continuum by the BEM}

In this part we briefly describe an implementation of the boundary element method to the solution of specific problems of underground continuum, for which the numerical method appears to be extraordinary advantageous.

The method, among others, reduces the problem by one. Further good application of the boundary element method is the optimization and/or contact problems which concern the boundary only. Then, in spite of the finite element method it suffices to study a change of location of boundary elements only.

The problem is solved as two-dimensional, i.e. a possible dislocation is long enough, and a narrow seam is considered. Moreover, the nonlinear behavior is considered in the region, which is sufficiently close to the dislocation, according to Mises theory. Suspicious dislocation is given from the experimental model from physically equivalent materials.

In our following consideration we will concentrate on the physically nonlinear problems (nonlinear evolution is also included in boundary conditions). Let us solve the problem in domain $\Omega$. We originate from the Cauchy equations:

$$
(\alpha+\mu) \frac{\partial}{\partial x_{i}} \operatorname{div} u+\mu \Delta u_{i}+X_{i}+\sum_{j} \frac{\partial \sigma_{i j}^{0}}{\partial x_{j}}=0, i=1, \ldots, 3
$$

where

$$
\operatorname{div} u=\frac{\partial u_{1}}{\partial x_{1}}+\frac{\partial u_{2}}{\partial u_{2}}, \quad \quad \Delta=\frac{\partial}{\partial x_{1}}+\frac{\partial}{\partial x_{2}}
$$

and $u=\left(u_{1}, u_{2}\right)$ is the displacement field, $\left(X_{1}, X_{2}\right)$ are components of the volume weight and $\sigma_{i j}{ }^{0}$ are components of the tensor of initial stress.

These equations will be solved in the coordinate system $0 x_{1}, x_{2}$. In the sense of BEM, (1) may be reformulated in an equivalent form:

$$
c_{k 1}(\xi) u_{1}(\xi)=\left(\varepsilon_{i j k}^{*}, \sigma_{i j}\right)+\left[p_{i k}, u_{i}\right]-\left\lfloor u_{i k}^{*}, p_{i}\right\rfloor-\left(u_{i k}^{*}, X_{i}\right)
$$

where [.] are boundary integrals, (.) are plane integrals, $c_{k l}$ is the matrix of coefficients depending on a position of $\xi, p$ is the vector of external forces, and quantities with an asterisk denote the relevant quantities of the fundamental solution.

From the Cauchy equations (respecting shearing stresses to be zero) we have the well known relations for a virgin state to get the initial stresses:

$$
\begin{gathered}
u_{1}^{o}=-\frac{1-2 v}{4 \mu(1-v)} X_{1} x_{1}^{2}+\text { const. }, \quad u_{2}^{o}=0 \\
\sigma_{1,1}^{o}=-X_{1} x_{1} \sigma_{1,2}^{o}=0, \sigma_{2,2}^{o}=-\frac{v}{1-v} X_{1} x_{1}
\end{gathered}
$$




\section{Contact problem}

Before we start the analysis preliminary considerations will be introduced. In order to explain the process of computation the two-dimensional problem will be treated. The three-dimensional problems are solved similarly.

Let the problem be described from experimental study. $\Omega^{\text {seam }}$ is the domain of the seam, for which the dislocation and the bearing capacity is to be assessed.

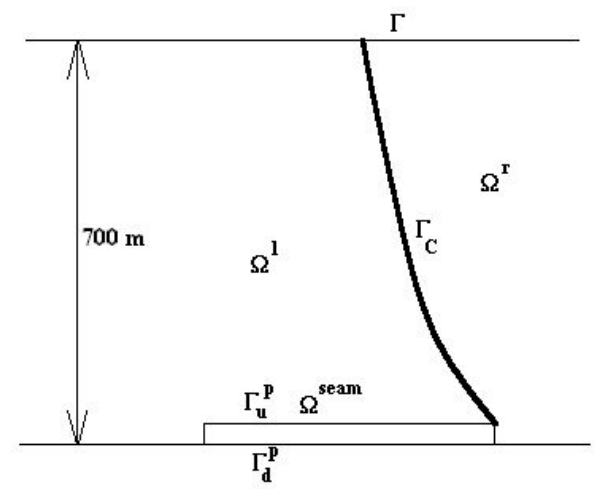

Figure 2: $\quad$ Domain and denotation of the example under study.

After discretization of (2) in the sense of the boundary element method the problem leads to the system of algebraic equations:

$$
\left[\begin{array}{lll}
H_{11} & H_{12} & H_{13} \\
H_{21} & H_{22} & A_{23} \\
H_{31} & H_{32} & A_{33}
\end{array}\right]\left\{\begin{array}{l}
u \\
u_{c}^{-} \\
u_{c}^{+}
\end{array}\right\}-\left[\begin{array}{lll}
G_{11} & G_{12} & G_{13} \\
G_{21} & G_{22} & G_{23} \\
G_{31} & G_{32} & G_{33}
\end{array}\right]+G_{11}^{g}\left\{\begin{array}{l}
g \\
g_{c}^{-} \\
g_{c}^{+}
\end{array}\right\}=\left\{\begin{array}{l}
F \\
F_{c}^{-} \\
F_{c}^{+}
\end{array}\right\}
$$

where the upper index - denotes "from the left" and + denotes "from the right", $\mathrm{g}$ is the vector of prescribed surface forces along the boundaries $\Gamma$ and $\Gamma_{p}, p_{c}$ is the vector of surface forces on fictitious contact $\Gamma_{c}$ and $F$ includes the effect of volume weight.

As the vector $g$ contains known quantities we can rearrange the previous equations to obtain:

$$
\left[\begin{array}{lll}
\mathrm{H}_{11} & \mathrm{H}_{12} & \mathrm{H}_{13} \\
\mathrm{H}_{21} & \mathrm{H}_{22} & \mathrm{~A}_{23} \\
\mathrm{H}_{31} & \mathrm{H}_{32} & \mathrm{~A}_{33}
\end{array}\right]\left\{\begin{array}{l}
\mathrm{u} \\
\mathrm{u}_{\mathrm{c}}^{-} \\
\mathrm{u}_{\mathrm{c}}^{+}
\end{array}\right\}-\left[\begin{array}{l}
\mathrm{G}_{12} \mathrm{p}_{\mathrm{c}}^{-}+\mathrm{G}_{13} \mathrm{p}_{\mathrm{c}}^{+} \\
\mathrm{G}_{21} \mathrm{p}_{\mathrm{c}}^{-}+\mathrm{G}_{23} \mathrm{p}_{\mathrm{c}}^{+} \\
\mathrm{G}_{31} \mathrm{p}_{\mathrm{c}}^{-}+\mathrm{G}_{33} \mathrm{p}_{\mathrm{c}}^{+}
\end{array}\right]=\left\{\begin{array}{l}
\mathrm{F}+\mathrm{G}_{11}^{\mathrm{g}} \\
\mathrm{F}_{\mathrm{c}}^{-}+\mathrm{G}_{21}^{\mathrm{g}} \\
\mathrm{F}_{\mathrm{c}}^{+}+\mathrm{G}_{31}^{\mathrm{g}}
\end{array}\right\}
$$


Suppose now that for example $u_{c}^{-}$and $u_{c}^{+}$is known. Then the problem is uniquely solvable, so that the matrix $\mathrm{H}_{11}$ is regular. For a similar reason the matrices $\mathrm{H}_{\mathrm{kk}}$ are regular, too. Also, the same assertion holds for the matrices $\mathrm{G}_{11 \text {, }}$ $i=1,2$. This is the general result of solvability of linear problems of elasticity by the boundary element method. We can conclude that the matrix $\mathrm{H}$ is singular, but the last submatrices are regular matrices. This is why it is possible to rearrange the system in the sense of matrix canonical transformations (in algorithm we use Gaussian elimination) to obtain:

$$
\left[\begin{array}{ccc}
\mathrm{H}_{11} & \mathrm{H}_{12} & \mathrm{H}_{13} \\
0 & \mathrm{~A}_{22} & \mathrm{~A}_{23} \\
0 & \mathrm{~A}_{32} & \mathrm{~A}_{33}
\end{array}\right]\left\{\begin{array}{c}
\mathrm{u} \\
\mathrm{u}_{\mathrm{c}}^{-} \\
\mathrm{u}_{\mathrm{c}}^{+}
\end{array}\right\}-\left\{\begin{array}{l}
\mathrm{B}_{11} \\
\mathrm{~B}_{21} \\
\mathrm{~B}_{31}
\end{array}\right\}\left\{\mathrm{P}_{\mathrm{c}}\right\}=\left\{\begin{array}{l}
\mathrm{C}_{11} \\
\mathrm{C}_{21} \\
\mathrm{C}_{31}
\end{array}\right\}
$$

where the balance condition

$$
p_{c}=p_{c}^{-}=-p_{c}^{+}
$$

was employed. The matrices are known while the vectors $\mathrm{u}$ and $\mathrm{p}$ remain unknown. From the last form the reducibility follows and we can employ the following system of equations:

$$
\begin{aligned}
& \mathrm{A}_{32} \mathrm{u}_{\mathrm{c}}^{-}+\mathrm{A}_{33} \mathrm{u}_{\mathrm{c}}^{+}-\mathrm{B}_{31} \mathrm{p}_{\mathrm{c}}=\mathrm{c}_{21} \\
& \mathrm{~A}_{22} \mathrm{u}_{\mathrm{c}}^{-}+\mathrm{A}_{23} \mathrm{u}_{\mathrm{c}}^{+}-\mathrm{B}_{21} \mathrm{p}_{\mathrm{c}}=\mathrm{c}_{22}
\end{aligned}
$$

Generally, along the contact line only balance condition holds and the compatibility is prescribed with the aid of more complicated relations. For example, suppose that at each nodal point along the contact line holds:

$$
\begin{gathered}
{[u]_{n}=u_{n}^{1}-u_{n}^{2} \geq 0} \\
\left|p_{t}\right| \leq T p_{n}+c \\
\left|p_{t}\right| \leq T p_{n}+c \Rightarrow E \lambda>0,[u]_{n}=-\lambda p_{t}
\end{gathered}
$$

where $T$ and $c$ are prescribed coefficients (they may very along the contact), $p_{n}$ and $p_{t}$ are projections of tractions to the normal and tangential direction with respect to the contact line, respectively. Then Uzawa's algorithm can be applied to the contact problem - see, e.g., [12]. In the domain the rheological model is applied according to Fig. 3 with the coefficients:

Elasticity modulus $E=10 \mathrm{GPa}$, Poisson's ratio $v=0.21$, peak cohesion = 1.2 $\mathrm{MPa}$, peak angle of internal fiction $=30^{\circ}$, dilation angle $=0^{0}$, peak tensile strength $=4 \mathrm{MPa}$ (not used), residua cohesion $=0.2 \mathrm{MPa}$, cohesion softening rate $=0.001$, residua fiction angle $=20^{\circ}$, fiction softening rate $=100$, rock viscosity $=10-19 \mathrm{MPa}$ (average used). 


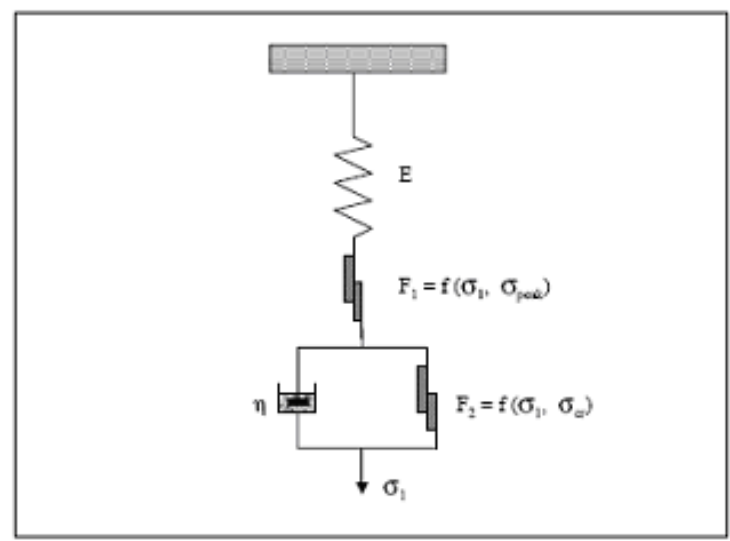

Figure 3: $\quad$ Schematic of the rheological model.

The critical shape of the searched dislocation is depicted in Fig. 2. The way of overall vertical stresses together with a description of typical longwall mining is illustrated in Fig. 4. Fig. 5 displays distribution of principal strain rates for two basic materials: the left describes a roof with plastic properties and the right material properties being "almost" brittle. The envelope of the peak stresses describes the most dangerous states for possible occurrence of bumps. The picture was obtained from numerical computation, based on tuned material properties from the scale model and in situ measurement of material parameters.

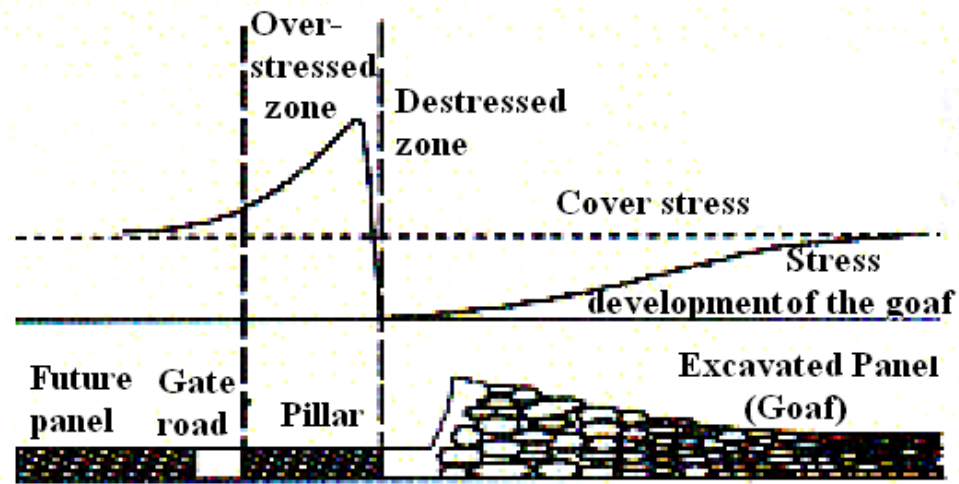

Figure 4: $\quad$ Stress state in the ground after the excavation of the panel.

\section{Conclusions}

Geotechnical data, mining experience, and long-term underground observations were analyzed in an effort to better understand causes of violent failure in U.S. coal mines. There it was shown that coal bumps are influenced by the interaction 
of geologic and stress conditions that govern the post failure behavior of coalmeasure rocks. Case studies provided new insight into the buildup of horizontal stresses, geometric factors that cause zones with high stress gradients, contrasts in stiffness and the mechanical properties of rock and seam, and failure of upper strata.

In this paper a combination of experimental tests in scale models from physically equivalent materials provided input data for numerical models, the mechanical properties which were tuned to get a more realistic view of the real situation. The aim of the numerical model was to determine critical dislocation, which causes the critical state on the interface of the overburden and coal seam. The energy accumulated at the tip can be calculated from the numerical model after finishing the computation using the above described conditions. The decisive factors are principal strain rates.

In any case, experience, numerical modeling, and engineering judgment should be used to assess the extent of yielding in mine roofs and floors; such controlled yielding may help reduce horizontal stresses and lead to less violent failures. Lack of yielding within the roof, coal, and seam promotes high bump potential where there is a high risk of a seismic event and a low factor of safety.
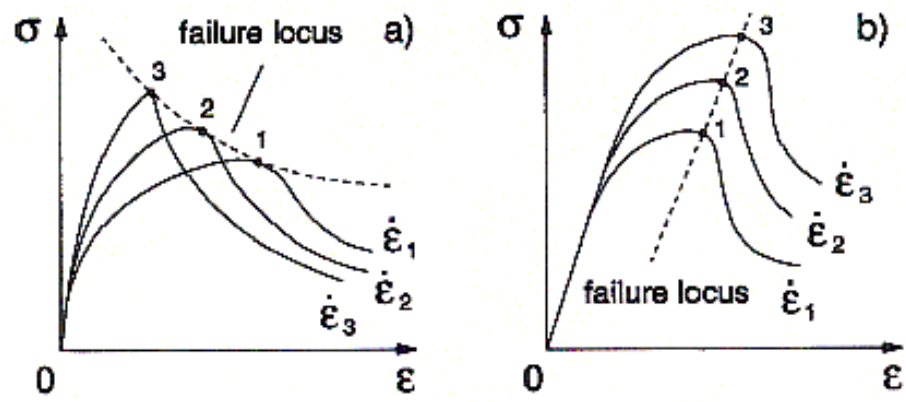

Figure 5: Two types of material response in compression for varying principal strain rates.

\section{Acknowledgements}

The first author has been financially supported by the Grant agency of the Czech Republic, project number 103/05/0679, and the second author was supported by the Grant agency of the Academy of the Czech Republic, project number IAA 2119402.

\section{References}

[1] Maleki, H., Jung, Y. \& Hollberg, K: Case Study of Monitoring Changes in Roof Stability. Int. J. Rock Mech. Min. Sci. and Geomech. Abstr., V. 30, No. 7, 1395-1401, 1993. 
[2] Elices, M., Guinea, G.V., Gomez, J. \& Planas, J. The cohesive zone model: advantages, limitations and challenges. Engineering Fracture Mechanics 69, 2002, 137-163.

[3] Haramy, K.H., \& McDonnell, J.P.: Causes and Control of Coal Mine Bumps. USBM RI 9225, 35 pp, 1988

[4] Haramy, K.Y., Magers, J.A. \& McDonnell, J.P. Mining under strong roof. 7th Int. Conf. on Ground Control in Mining, Bureau of Mines, Denver, USA, 1992, 179-194.

[5] Haramy, K.Y. \& Brady, B.T. A methodology to determine in situ rock mass failure. Internal report of Bureau of Mines, Denver, CO, USA, 1995.

[6] Mark, C.: Pillar Design Methods for Longwall Mining. USBM IC 9247, 52 p., 1990.

[7] Kuch, R., Lippmann, H. \& Zhang, J. Simulating coal mine bumps with model material. Rockbursts and seismicity in mines, Gibowitz \& Lasocki (eds.), Balkema, Rotterdam, 1997, 23-25.

[8] Babuska, I., Melenk, J.M.: The partition of unity method, Int. J. Numer. Meth. Engrg. 40 (1997) 727-758

[9] Lin, J.S.: A mesh-based partition of unity method for discontinuity modeling, Comput. Meth. Appl. Mech. Engrg. 192 (2003) 1515-1532

[10] Chen, G., Ohnoshi, Y., Ito, T. Development of high-order manifold method, Int. J. Numer. Meth. Engrg. 43 (1998) 685-712

[11] Procházka, P. Application of discrete element methods to fracture mechanics of rock bursts. Engng. Fract. Mech. 2003.

[12] Dolezel, V. \& Prochazka, P.: Characterization of dislocations in underground mass using coupled modeling. CMEM, Portland, Maine, USA, 2005, 333-341 